\title{
Thermally activated inter-dots carriers' transfer in InAs QDs with InGaAs underlying layer: Origin and dependence on the post-growth intermixing
}

\author{
B. Ilahi a, b, ${ }^{*}$, O. Nasr ${ }^{\text {b }}$, B. Paquette ${ }^{\text {c }}$, M.H. Hadj Alouane ${ }^{\text {b }}$, N. Chauvin ${ }^{\text {d }}$, B. Salem ${ }^{\text {e, f }}$, L. Sfaxi ${ }^{\text {b }}$, C. Bru-Chevalier ${ }^{\text {d }}$, \\ D. Morris g, R. Ares g, V. Aimez ${ }^{g}, H$. Maaref ${ }^{b}$ \\ ${ }^{a}$ King Saud University, Department of Physics \& Astronomy, College of Sciences, 11451, Riyadh, Saudi Arabia \\ ${ }^{\mathrm{b}}$ Universite de Monastir, Laboratoire de Micro-Optoelectronique et Nanostructures (LMON), Faculte des Sciences, Avenue de \\ l'environnement, 5019, Monastir, Tunisia \\ c NRC of Canada, Canadian Photonics Fabrication Centre, 1200, Montreal Rd., Ottawa, K1A 0R6, Canada \\ d Universite de Lyon, Institut des Nanotechnologies de Lyon (INL)-UMR5270-CNRS, INSA-Lyon, 7 avenue Jean Capelle, 69621, \\ Villeurbanne, France \\ e Univerite de Grenoble Alpes, LTM, F-38000, Grenoble, France \\ ${ }^{\mathrm{f}}$ CNRS, LTM, F-38000, Grenoble, France \\ g Institut Interdisciplinaire d'Innovation Technologique (3IT), and Laboratoire Nanotechnologies Nanosystemes (LN2)-CNRS UMI-3463, \\ Universitede Sherbrooke, Sherbrooke, QC, Canada
}

\begin{abstract}
This paper reports on experimental and theoretical investigation of atyical temperature-dependent photoluminescence properties of InAs quantum dots in close proximity to InGaAs strain-relief underlying quantum well. The impact of a post-growth intermixing process on these properties has been studied. For the as-grown sample, the maximum of the emission band follows a sigmoidal function while the photoluminescence linewidth mimics a $\mathrm{V}$-shape function as the temperature increases, from 11 to $300 \mathrm{~K}$. These behaviors are attributed to thermally activated carrier transfer mechanisms within the inhomogenious distribution of quantum dots. These atypical behaviors are found to disappear progressively with the degree of intermixing and consequent narrowing of the dot size dispersion. The experimental results have been interpreted in the frame of the localized states ensemble model revealing that the large dots size distribution is the main origin of the observed anomalies. Furthermore, the calculations show that the quantum well continuum states act as a transit channel for the redistribution of thermally activated carriers.
\end{abstract}

\section{Introduction}

Self organized semiconductor InAs/GaAs QDs have been a subject of intense research activity due to their potential application in the improvement of future photovoltaic [1] and optoelectronic devices [2-4]. However, as a direct consequence of the Stranski-Krastanow (SK) growth mode, the optimal exploitation of these QDs are seriously limited by several parameters, such as low surface density [5], strain accumulation [6], size distribution [7]. Several attempts have been made to improve the QDs properties including the use of strain reducing layers [8,9], strain

\footnotetext{
* Corresponding author. King Saud University, Department of Physics \& Astronomy, College of Sciences, 11451, Riyadh, Saudi Arabia.

E-mail address: bilahi@ksu.edu.sa (B. Ilahi).
}

compensation [10], post growth intermixing [11], and surfactant [12]. Furthermore, understanding the temperature dependent photoluminescence mechanism is critical to optimize QDs devices that function at room temperature. Indeed the anomalous temperature-dependent luminescence properties, due to the thermally activated inter-dots carriers transfer, seems to be induced by the QDs' self-organization nature [13-15]. Consequently, the emission energy and the luminescence linewidth have been respectively shown to follow a sigmoidal function of and a $\mathrm{V}$-shape variation [16-21]. However, the reported studies were focused on as-grown QDs and less attention has been made for the investigation of this thermally induced carriers process dependence on the post-growth intermixing.

In this context, we report on the impact of the intermixing degree on the temperature dependent sigmoidal emission energy and 
V-shaped PL linewidth observed for InAs QDs with InGaAs underlying layer. The origin of the carrier's thermally activated transfer mechanisms and its dependence on the QDs size dispersion is discussed using a model describing the steady-state luminescence from localized states ensemble (LSE) [22].

\section{Experimental details}

The investigated sample consists of a single InAs QDs layer with $10 \mathrm{~nm} \operatorname{In}_{0.15} \mathrm{Ga}_{0.85}$ As strain reducing underlying layer, grown by molecular beam epitaxy on a Si-doped (001) GaAs substrate. The InAs nominal thickness was 2.4 monolayers, deposited at a growth rate of $0.08 \AA / \mathrm{s}$ and followed by 30 s growth interruption under As flux prior to the deposition of $50 \mathrm{~nm}$ thick GaAs cap layer. The InGaAs and GaAs materials are deposited respectively at $0.54 \AA / \mathrm{s}$ and $2.4 \AA / s$. The structure is schematically presented by Fig. 1 . Additionally, $10 \mathrm{~nm}$ thick $\operatorname{In}_{0.15} \mathrm{Ga}_{0.85}$ As quantum well has been grown as a reference sample.

Besides, five pieces, cut from the central region of the as grown sample, have been used to prepare samples with different degree of post growth intermixing by rapid thermal annealing. The RTA process was performed at temperature from $650{ }^{\circ} \mathrm{C}$ to $800{ }^{\circ} \mathrm{C}$ for $30 \mathrm{~s}$ in a nitrogen ambient atmosphere. During annealing, the samples were protected from desorption of As atoms by semiinsulating GaAs proximity cap.

The PL measurements were performed using a conventional lock-in technique. The samples were mounted in a closed cycle temperature controlled He cryostat. The PL emission was detected by a nitrogen cooled silicon CCD detector coupled to a monochromator.

\section{Results and discussion}

\subsection{PL properties at $11 \mathrm{~K}$}

The normalized 10K PL spectra recorded at different excitation power from the as-grown sample are shown in Fig. 2a. The state filling effect is clearly observed through the increase of the QDs excited states PL peak's intensity with increasing the excitation power. It's also interesting to mention the appearance of an emission peak at $1.354 \mathrm{eV}$ which is attributed to the InGaAs QW/underlying layer. The later attribution has been confirmed by photoluminescence of excitation (not shown here) and photoreflectance spectroscopy [23]. Previous investigation of this structure revealed that the indium segregation induces an increase in both QDs surface density and size dispersion leading to the observed broadening of the PL spectra [23]. This structure has been subjected to post-growth rapid thermal annealing during $30 \mathrm{~s}$ at temperature ranging from $650{ }^{\circ} \mathrm{C}$ to $800{ }^{\circ} \mathrm{C}$. The corresponding PL

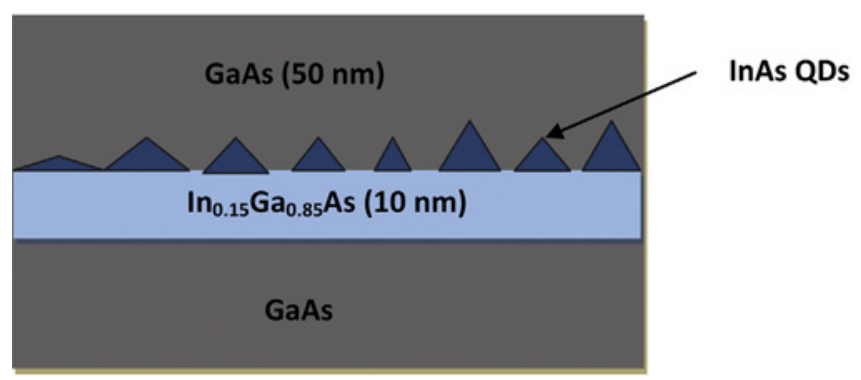

Fig. 1. Schematic presentation of the investigated structure containing InAs QDs with InGaAs underlying layer. spectra are shown in Fig. 2b. Noticeable effect of the intermixing has been observed for annealing temperature higher than $650{ }^{\circ} \mathrm{C}$, where the QDs emission energy show a blue-shift by up to $100 \mathrm{meV}$. Furthermore, the PL linewidth shows a monotonous reduction with increasing of the intermixing degree from $61 \mathrm{meV}$ to $37 \mathrm{meV}$ (Fig. 2c).

These results give clear evidence of the homogenization of the QDs size dispersion. Indeed, the atomic intermixing alters the QDs composition resulting in a change of the carriers' confinement potential, emission energy and size dispersion [24-26]. It's worth noting that the increase of the intermixing induced a decrease of the PL integrated intensity testifying also an enhancement of the nonradiative defects density [24].

\subsection{PL as a function of temperature}

The temperature dependence of the ground state PL peak energy and their corresponding FWHM is shown in Fig. 3 for both the as-grown and the intermixed QD samples. The temperature dependent PL properties of the as grown QDs exhibit a sigmoidal emission energy characterized by a fast redshift in the $50-200 \mathrm{~K}$ range. Meanwhile, the PL FWHM decreases first in the low temperature range and then increases again ( $\mathrm{V}$-shaped variation). This atypical variation has not been observed, neither for reference InAs QDs in GaAs matrix nor for QDs with strain reducing capping layer grown in the same conditions and showing narrower size distribution and lower surface density [24]. This makes the observed phenomena likely to originate from the large dots size dispersion.

As stated in the previous section, an improvement of the QDs size uniformity with an increasing intermixing has been observed. Accordingly, we note that the deviation from the Varshni empirical law for bulk InAs material gets reduced with increasing intermixing. Despite the extinction of the PL spectra at relatively low temperature, the sigmoidal variation has been considerably reduced at high annealing temperature. The observed behavior can be interpreted by the carriers activation and transfer between the inhomogeniously distributed QDs. For the highest annealing temperature $\left(800{ }^{\circ} \mathrm{C}\right)$ the QDs emission energy is around $50 \mathrm{meV}$ below that of the QW. The shallow barrier energy may facilitate the carriers thermal escape at relatively low temperature. Additionally the high intermixing degree could also generate a high non radiative defects density. For these reasons, the QDs PL quenches around $100 \mathrm{~K}$.

For more quantitative interpretation aiming to illuminate the carriers transfer mechanism responsible for the observed behavior we have employed the LSE model [22]. This model treats the QDs ensemble as a system of Gaussian distributed localized states allowing derivation of quantitative information such as the localized states broadening parameter and the activation energy. The first parameter can be linked to the QDs size dispersion [27] and the second one allows the identification of the carriers transfer channel [28]. In this model, the photogenerated carriers in the barrier material are captured by the QDs states prior to their recombination. The captured carriers are localized in the QDs states at low temperature. However, as the temperature increases, the carriers can be thermally activated from shallow states to a mediator channel before being recaptured by the deeper states (larger QDs).

According to this model, the temperature-dependent PL peak energy can be expressed as follow [22]:

$E(T)=E_{0}-\alpha \frac{T^{2}}{\beta+T}-x(T) \cdot k_{B} T$,

where $\alpha$ is the Varshni parameter and $\beta$ the Debye temperature [29] taken to be around $0.25 \mathrm{meV} \mathrm{K}^{-2}$ and $180 \mathrm{~K}$, respectively. The extra 

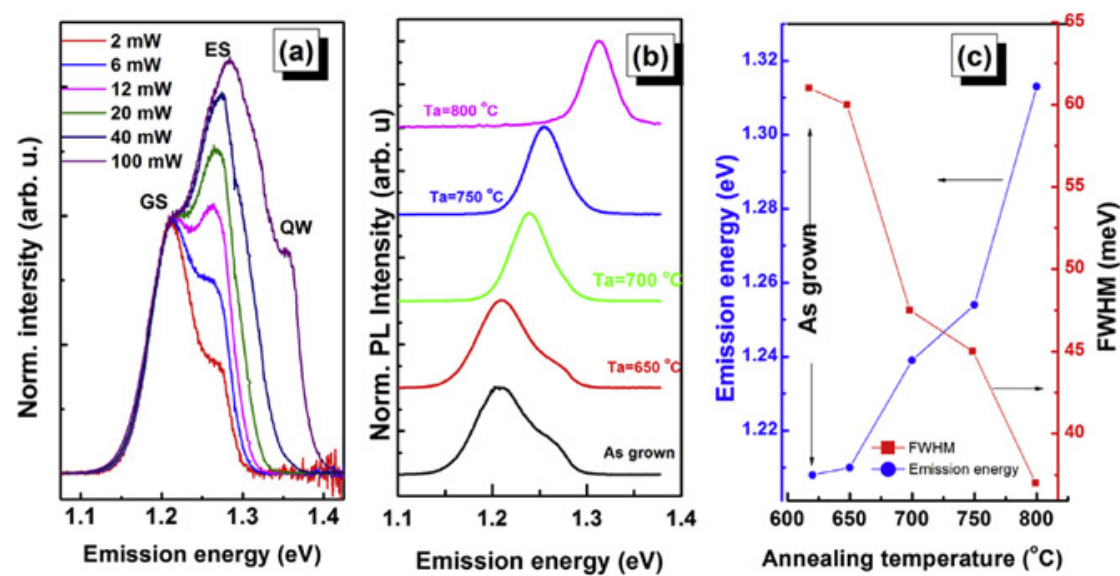

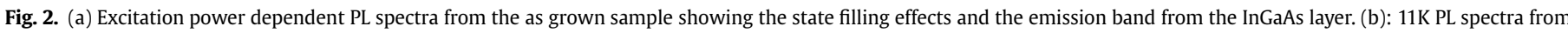

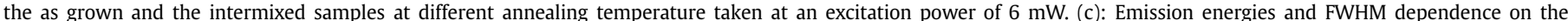
intermixing degree.
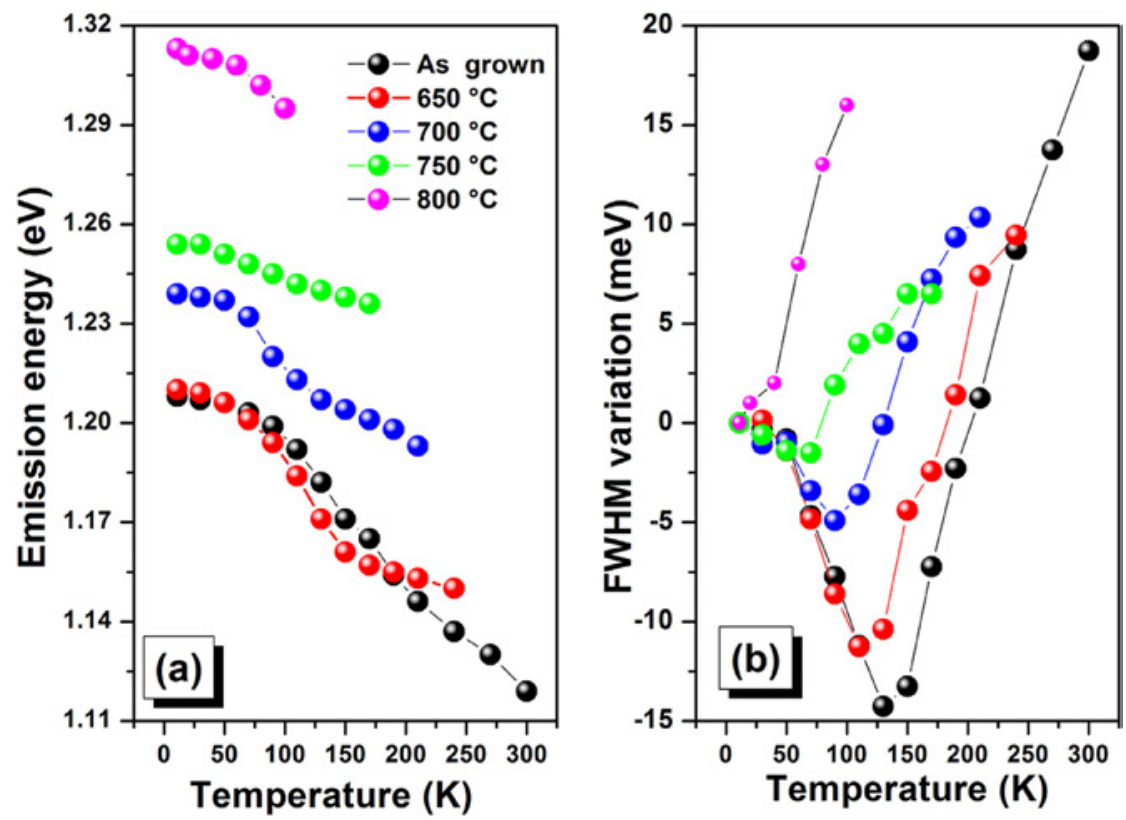

Fig. 3. Temperature dependence of the emission energies and PL FWHM. (a) Emission energies and (b) PL FWHM variation with respect to their values at 11 K.

term $\left(x(T) \cdot k_{B} T\right)$ describes the deviation of the PL peak's maximum from the expected bandgap shrinkage from Varshni's empirical relation. The dimensionless term $x(T)$ can be evaluated by numerically solving the following nonlinear equation [22]:

$x(T) \cdot e^{x(T)}=\left[\left(\frac{\sigma}{k_{B} T}\right)^{2}-x\right]\left(\frac{\tau_{r}}{\tau_{t r}}\right) e^{-E_{a} / k_{B} T}$,

where $\sigma$ is the standard deviation of the localized states distribution. $1 / \tau_{\mathrm{tr}}$ and $1 / \tau_{\mathrm{r}}$ are respectively, the escape rate and the radiative recombination rate taken as constant and characteristics of each sample. The energy $E_{a}$ corresponds to the energy barrier that the carriers have to overcome to be thermally excited to the mediator energy level and subsequently redistribute by recapture process.

The variation of the PL linewidth with temperature is described within the LSE model as the convolution of a function describing the shape of the luminescence spectrum [22] with a Lorentzian function that takes into account the thermal broadening related to phonon and impurity scattering mechanisms given by:

$$
\left(4[E(T)]^{2}+\left[\Gamma_{0}+\Gamma_{a c} T+\Gamma_{L O} \frac{1}{\exp \left(\frac{E_{L O}}{k_{B} T}\right)-1}\right]^{2}\right)^{-1}
$$

where $\Gamma_{0}$ is the intrinsic linewidth at $11 \mathrm{~K}, \Gamma_{\mathrm{ac}}$ and $\Gamma_{\mathrm{LO}}$ correspond to the scattering coefficients related to the interaction of carriers with acoustic and longitudinal optical (LO) phonons [30,31], $\mathrm{E}_{\mathrm{LO}}$ is the LO phonon energy, these parameters are taken to be $50 \mu \mathrm{eV} / \mathrm{K}$, $35 \mathrm{meV}$, and $30 \mathrm{meV}$ respectively. More details on this model can be found elsewhere [22,28,32].

The fitting curves for the emission energies and the PL FWHM with temperature from the investigated samples as well as the experimental data are given by Fig. 4. The optimum parameters yielding the best fit of the experimental data are listed in Table 1. 

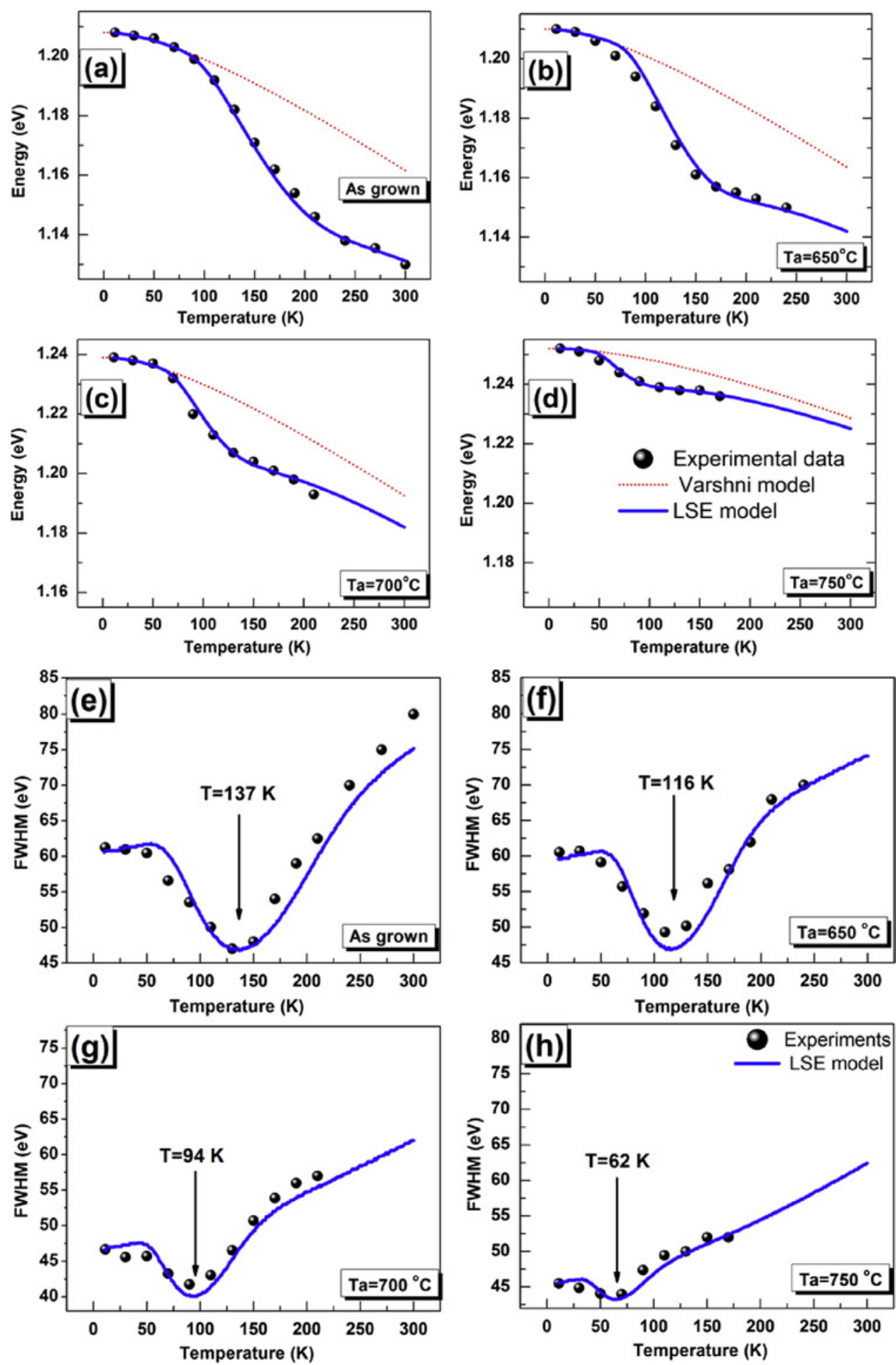

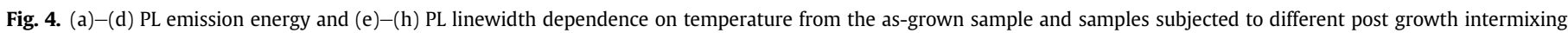

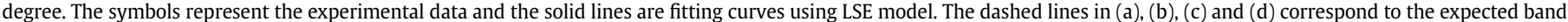
gap shrinkage by Varshni law using the above mentioned parameters except for $\mathrm{Ta}=750{ }^{\circ} \mathrm{C}$ where $\beta=370 \mathrm{~K}$.

The LSE model succeeds in accurate reproducing of the atypical PL properties variation with temperature.

The broadening parameter $(\sigma)$ is found to decrease with increasing annealing temperature in accordance with the $11 \mathrm{~K}-\mathrm{PL}$ linewidth shrinkage (Fig. 2c) confirming the improvement of the QDs size dispersion with increasing intermixing. The variation of the PL FWHM with temperature appears to be governed by the carrier thermal activation and redistribution. Indeed when the temperature increases, the carriers get delocalized from the shallower states (small QDs) and recaptured by larger QDs. In this stage, an increasing number of small size QDs no longer contribute to the luminescence leading to the decrease of the PL linewidth and fast 
Table 1

Parameters yielding the best fit of the experimental emission energies and PL FWHM by the LSE model.

\begin{tabular}{llll}
\hline Annealing temperature $\left({ }^{\circ} \mathrm{C}\right)$ & $\sigma(\mathrm{meV})$ & Ea $(\mathrm{meV})$ & $\tau_{\mathrm{tr}} / \tau_{\mathrm{tr}}$ \\
\hline As-grown & 28 & 110 & $9.5 \times 10^{-2}$ \\
650 & 24 & 102 & $6.7 \times 10^{-2}$ \\
700 & 16.6 & 77 & $4.5 \times 10^{-2}$ \\
750 & 9 & 53 & $1.3 \times 10^{-2}$ \\
\hline
\end{tabular}

redshift of the emission energies. At a given characteristic temperature, the PL properties become dominated by the electronphonon scattering leading to a monotonic increase of the PL linewidth and slower decreasing rate of the emission energy. The characteristic temperature (indicated by arrows in Fig. 4 (e)-(h)), qualitatively marking the end of the carriers redistribution, is found to decrease with increasing intermixing. This indicates that less thermal energy is required for carriers delocalization as the intermixing increases.

Accordingly, the calculated activation energy is also found to decrease with increasing annealing temperature. This behavior indicates that the effective energy barrier that carriers need to overcome to be delocalized is lowered by the intermixing process. This phenomenon is a direct consequence of the atomic intermixing and consequent QDs compositional change leading to the decrease of the carriers confinement potential and reducing of the size dispersion. Additionally, the time ratio constant $\tau_{\mathrm{tr}} / \tau_{\mathrm{r}}$, is found to decrease with increasing intermixing. The observed reduction is most likely dominated by the increased escape rate as a consequence of intermixing induced lowering of the confining potential energy.

To identify the carriers transfer channel, we considering that within the present conditions, the QW remain insensitive to the intermixing process. Accordingly, the activation energy $\left(\mathrm{E}_{\mathrm{a}}\right)$ and the difference between the emission energy from the QW and that from the QDs ground states ( $\mathrm{E}_{\mathrm{QW}}-\mathrm{E}_{\mathrm{QDs}}$ ) have been plotted in Fig. 5 against the annealing temperature. Furthermore, as shown by the inset of Fig. 5, PL spectra from a reference QW identical to the underlying layer have been recorder for different annealing temperature revealing that the QW emission energy does not show any

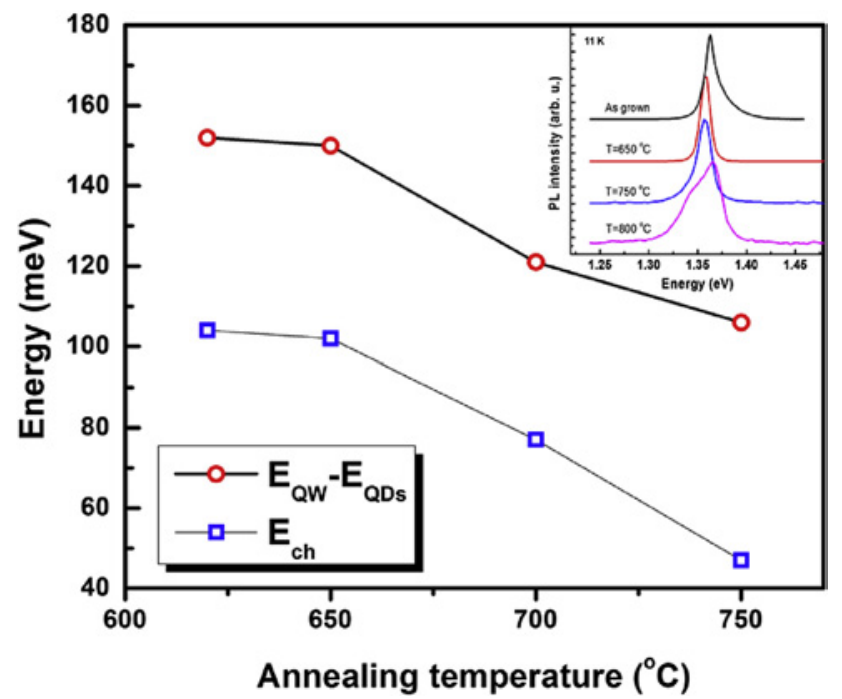

Fig. 5. Comparative plot of the calculated activation energy (empty squares) and the difference between the QW transition energy and that of the QDs ground states at $11 \mathrm{~K}$ (empty circles). The inset show the evolution of the $11 \mathrm{~K}$ PL spectra from a reference QW sample subjected to the same annealing conditions.

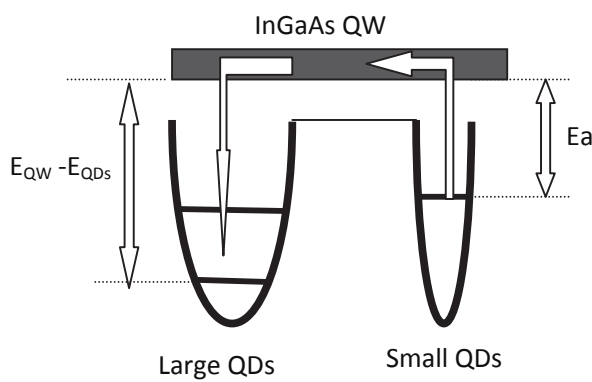

Fig. 6. Simplified schematic presentation relative to one type of carrier activation from small size QDs and subsequent recapture by bigger dots size.

significant shift. This result strengthens our interpretation based on the insensitivity of the QW emission energy to the annealing conditions adopted in this work.

Both quantities show a monotonic decrease for annealing temperature higher than $650{ }^{\circ} \mathrm{C}$. Furthermore, the activation energy is approximately $40 \mathrm{meV}$ below the $\mathrm{QW}$ continuum states regardless of the intermixing degree. Such a value is close to the $11 \mathrm{~K}-\mathrm{PL}$ FWHM. Accordingly, the calculated energy barrier can easily be matched to the energy difference between the small size QDs' emission energy and that of the QW continuum states as schematically presented by Fig. 6 .

Therefore, the QW continuum states in the present case serves as mediator for thermally activated carriers redistribution within the broadened QDs states responsible for the observed anomalies in the temperature dependent PL properties.

\section{Summary}

The atypical temperature dependent PL properties, shown by InAs QDs with InGaAs underlying layer has been investigated as a function of the post-growth intermixing degree. The observed anomalies are found to arise from the large size dispersion. The sigmoidal emission energy and V-shaped PL peak's FWHM variation is shown to be reduced with increasing annealing temperature and tends to the usual behavior at high intermixing degree. The QW continuum states have been identified as the mediator of thermally activated carrier redistribution. These results help to improve the understanding of the temperature-dependent carrier dynamics in strain-engineered QDs.

\section{Acknowledgment}

The first author would like to extend his sincere appreciation to the Deanship of Scientific Research at king Saud University for funding this work through the Research group NO: RG -1436-014.

\section{References}

[1] K.A. Sablon, J.W. Little, V. Mitin, A. Sergeev, N. Vagidov, K. Reinhardt, Strong enhancement of solar cell efficiency due to quantum dots with built-in charge, Nano Lett. 11 (6) (2011) 2311.

[2] L.V. Asryan, M. Grundmann, N.N. Ledentsov, O. Stier, R.A. Suris, D. Bimberg, Maximum modal gain of a self-assembled InAs/GaAs quantum-dot laser J. Appl. Phys. 90 (2001) 1666.

[3] T. Kita, M. Suwa, T. Kaizu, Y. Harada, Polarization-insensitive optical gain characteristics of highly stacked InAs/GaAs quantum dots, J. Appl. Phys. 115 (2014) 233512.

[4] S. Maimon, E. Finkman, G. Bahir, S.E. Schacham, J.M. Garcia, P.M. Petroff, Intersublevel transitions in InAs/GaAs quantum dots infrared photodetectors, Appl. Phys. Lett. 73 (1998) 2003.

[5] S. Fretheim Thomassen, T. Worren Reenaas, B.O. Fimland, InAs/GaAs quantum dot density variation across a quarter wafer when grown with substrate rotation, J. Cryst. Growth 323 (2011) 223.

[6] J. Zribi, B. Ilahi, D. Morris, V. Aimez, R. Arès, Chemical beam epitaxy growth 
and optimization of InAs/GaAs quantum dot multilayers, J. Cryst. Growth 384 (2013) 21.

[7] Alexana Roshko, Todd E. Harvey, Brit L. Hyland, Susan Y. Lehman, Keith D. Cobry, Lateral variations in self-assembled InGaAs quantum dot distributions, J. Cryst. Growth 311 (2009) 4109.

[8] B. Ilahi, L. Sfaxi, G. Bremond, H. Maaref, Tuning optical properties of high in content InGaAs/GaAs capped InAs quantum dots by post growth rapid thermal annealing, Mater. Sci. Eng. C 26 (5) (2006) 971.

[9] S. Li, J. Bi, M. Li, M. Yang, M. Song, G. Liu, W. Xiong, Y. Li, Y. Fang, C. Chen, G. Lin, W. Chen, C. Wu, D. Wang, Investigation of GaInAs strain reducing layer combined with InAs quantum dots embedded in $\mathrm{Ga}(\mathrm{In})$ As subcell of triple junction GaInP/Ga(In)As/Ge solar cell, Nanoscale Res. Lett. 10 (2015) 111.

[10] X.Q. Zhang, S. Ganapathy, I. Suemune, H. Kumano, K. Uesugi, Yoichi Nabetani, Takashi Matsumoto, Improvement of InAs quantum-dot optical properties by strain compensation with GaNAs capping layers, Appl. Phys. Lett. 83 (2003) 4524.

[11] A. Babiński, J. Jasiński, R. Bożek, A. Szepielow, J.M. Baranowski, Rapid thermal annealing of InAs/GaAs quantum dots under a GaAs proximity cap, Appl. Phys. Lett. 79 (2001) 2576.

[12] H. Zhao, A. Malko, Z.H. Lai, Effect of bismuth on structural and electrical properties of InAs films grown on GaAs substrates by MBE, J. Cryst. Growth 425 (2015) 89.

[13] V.G. Dorogan, Y.I. Mazur, J.H. Lee, Z.M. Wang, M.E. Ware, G.J. Salamo, Therma peculiarity of AlAs-capped InAs quantum dots in a GaAs matrix, J. Appl. Phys. 104 (2008) 104303.

[14] T.V. Torchynska, J.L. Casas Espinola, L.V. Borkovska, S. Ostapenko, M. Dybiec, O. Polupan, N.O. Korsunska, A. Stintz, P.G. Eliseev, K.J. Malloy, Thermal activation of excitons in asymmetric InAs dots-in-a-well InxGa ${ }_{1-x} A s / G a A s$ structures, J. Appl. Phys. 101 (2007) 024323.

[15] C. Lobo, R. Leon, S. Marcinkecicius, W. Yang, P.C. Sercel, X.Z. Liao, J. Zou, D.J. H, Cockayne, inhibited carrier transfer in ensembles of isolated quantum dots, Phys. Rev. B 60 (1999) 16647.

[16] C. Lobo, R. Leon, S. Marcinkevicius, W. Yang, P.C. Sercel, X.Z. Liao, J. Zou, D.J.H. Cockayine, Inhibited carrier transfer in ensembles of isolated quantum dots, Phys. Rev. B 60 (24) (1999) 16647.

[17] S. Sanguinetti, M. Henini, M. Grassi Alessi, M. Capizzi, P. Frigeri, S. Franchi Carrier thermal escape and retrapping in self-assembled quantum dots, Phys. Rev. B 60 (11) (1999) 8276.

[18] T.-E. Nee, Y.-F. Wu, R.-M. Lin, Effect of carrier hopping and relaxing on photoluminescence line shape in self-organized InAs quantum dot heterostructures, J. Vac. Sci. Technol. B 23 (3) (2005) 954.

[19] T.-E. Nee, Y.-F. Wu, C.-C. Cheng, H.-T. Shen, Carrier dynamics study of the temperature and excitation-dependent photoluminescence of InAs/GaAs quantum dots, J. Appl. Phys. 99 (1) (2006) 013506.

[20] G. Muñoz-Matutano, I. Suárez, J. Canet-Ferrer, B. Alén, D. Rivas, Size dependent carrier thermal escape and transfer in bimodally distributed self assembled InAs/GaAs quantum dots, J. Appl. Phys. 111 (2012) 123522.

[21] B. Ilahi, L. Sfaxi, H. Maaref, G. Bremond, G. Guillot, Long wavelength vertically stacked InAs/GaAs (001) quantum dots with a bimodal size distribution: optical properties and electronic coupling, Superlattices Microstruct. 36 (1) (2004) 55.

[22] Q. Li, S.J. Xu, M.H. Xie, S.Y. Tong, A model for steady-state luminescence of localized-state ensemble, Europhys. Lett. 71 (2005) 994.

[23] O. Nasr, M.H. Hadj Alouane, H. Maaref, F. Hassen, L. Sfaxi, B. Ilahi, Comprehensive investigation of optical and electronic properties of tunable InAs QDs optically active at O-band telecommunication window with (In)GaAs surrounding material, J. Lumin. 148 (2014) 243.

[24] O. Nasr, M.H. Hadj Alouane, B. Ilahi, B. Salem, L. Sfaxi, H. Maaref, Postgrowth intermixing of strain engineered InAs/GaAs quantum dots, J. Alloys Compd. 615 (2014) 683.

[25] T.M. Hsu, Y.S. Lan, W.-H. Chang, N.T. Yeh, J.-I. Chyi, Tuning the energy levels of self-assembled InAs quantum dots by rapid thermal annealing, Appl. Phys. Lett. 76 (2000) 691.

[26] B. Ilahi, Z. Zaâboub, B. Salem, D. Morris, V. Aimez, L. Sfaxi, H. Maaref, Intermixing of InAs/GaAs quantum dots by proton implantation and rapid thermal annealing, Mater. Sci. Semicond. Process 12 (2009) 71.

[27] Q. Li, S. Barik, H.H. Tan, C. Jagadish, Effect of ion-implantation enhanced intermixing on luminescence of InAs/InP quantum dots, J. Phys. D Appl. Phys. 41 (2008) 205107

[28] Q. Li, S.J. Xu, M.H. Xie, S.Y. Tong, Origin of the 'S-shaped' temperature dependence of luminescent peaks from semiconductors, J. Phys. Condens. Matter 17 (2005) 4853.

[29] Y.P. Varshni, Temperature dependence of the energy gap in semiconductors, Phys. Amst. 34 (1967) 149.

[30] M.B. Smirnov, V.G. Talalaev, B.V. Novikov, S.V. Sarangov, G.É. Tsyrlin, N.D. Zakharov, Numerical simulation of the temperature dependence of the photoluminescence spectra of InAs/GaAs quantum dots, Phys. Solid State 49 (2007) 1184

[31] V.G. Talalaev, B.V. Novikov, M.B. Smirnov, V.V. Kachakanov, G. Gobsch, R. Golhahhn, A. Winzer, G.E. Cirilin, V.A. Egorov, V.M. Ustinov, Photoluminescence of isolated quantum dots in metastable InAs arrays, Nanotechnology 13 (2002) 143.

[32] M.H. Hadj Alouane, A. Helali, D. Morris, H. Maaref, V. Aimez, B. Salem, M. Gendry, B. Ilahi, Carriers' localization and thermal redistribution in post growth voluntarily tuned quantum dashes' size/composition distribution, J. Lumin. 145 (2014) 595. 